\title{
Cloning and Expression Analysis of CER1 in Blackberry (Rubus Spp.)
}

\author{
Xun-ju LIU ${ }^{1, \#}$, Yan WANG ${ }^{2, \#}$, Hao-ru TANG ${ }^{1}$ and Qing CHEN $^{1, a, *}$ \\ ${ }^{1}$ College of Horticulture, Sichuan Agricultural University, Chengdu 61130, Sichuan, \\ China \\ ${ }^{2}$ Institute of Pomology and Olericulture, Sichuan Agricultural University, Chengdu \\ 611130, Sichuan, China \\ "These authors contributed equally to this work \\ asupnovel@gmail.com \\ *Corresponding author
}

Keywords: Blackberry, CER1, Cloning and expression, Light irradiation

\begin{abstract}
To understand the molecular mechanisms of cuticle wax formation in blackberry, the waxy synthetic related gene CERI was cloned from blackberry. The open reading frame of this gene was $1878 \mathrm{bp}$, encoding 625 amino acid residues with a molecular weight of $72.3 \mathrm{kD}$ and a theoretical isoelectric point of 8.59. Its amino acid sequence has the highest identity with strawberry. The results of real-time quantitative PCR showed that the gene expression increased with the maturation of blackberry. With the increase of the ratio of red or blue light, the expression of CERI gene firstly decreased and then increased. Under the condition of red and blue light at a ratio of 2:8, the expression of CER1 was the lowest. The reason may be related to the different mechanisms of photoreceptor response to red and blue light.
\end{abstract}

\section{Introduction}

Cuticle is a layer of hydrophobic protective coating laying on the surface of terrestrial plants in direct contact with the air. The stratum corneum wax consists of the outer wax and the inner wax which are deeply embedded in the stratum corneum. The components of the stratum corneum are mainly linear aliphatic and its derivatives, as well as some terpenoids and flavonoid secondary metabolites [1]. Plant epidermis wax plays an important role in plant adaptation to biotic stresses and abiotic stresses. It limits plant non-stomatal transpiration and improves plant drought resistance. It also helps plants to clean themselves, reducing dust, pollen and spore attachment; preventing plants from being exposed to excessive UV radiation. It is also play a key role in plant-pathogenic bacteria and plant-insect interactions. The integrity of cuticle layer can ensure the normal demarcation of plant organs to prevent plant organ fusion [2].

With the LACS gene in Arabidopsis genome [3], the OSC gene in tomato [4] and many other genes related to wax synthesis have been cloned. The plant epidermal wax synthesis pathway has been basically clarified. The $\mathrm{C} 16$ and $\mathrm{C} 18$ fatty acid precursors in the endoplasmic reticulum are transformed into long-chain fatty acids, and then through the decarbonylation pathway and acylation reduction into a variety of waxy products, including alcohols, aldehydes, and ketones [5, 6]. Among these steps, the decarboxylation pathway is a key step in the process of waxy synthesis due to the observed high content of paraffin and secondary alcohol in plant epidermis. Arabidopsis thaliana eceriferum (CERl) gene, encoding a fatty aldehyde decarbonylase, 
located in the endoplasmic reticulum, is a key enzyme in the decarbonylation pathway [7]. In addition, CER1 is also involved in the decarbonylation pathway in other species such as rice, tomato and wheat [8]. However, the current reports on the specific mechanism of CERI and its regulatory mechanisms are extremely limited. More comprehensive experimental exploration is needed, especially on the molecular mechanism of wax metabolism regulation.

Many of the wild resources of Rubus plant fruit surface and the stems are covered with a thick waxy layer, which is highly resistant to sun light. However, there are very few cultivars of raspberry cultivars that have such characteristics. Therefore, preventing fruit from sunburn is still one of the measures that must be taken in the fruit production. In this study, the spineless blackberry 'Arapaho' was used as a material. From the transcriptomic data from previous research in our group, the transcript sequence homologous to Arabidopsis CERl was extracted. Gene-specific primers were designed and the $C E R 1$ gene was cloned by RT-PCR. The sequence of $C E R I$ gene in blackberry was analyzed by bioinformatics method. The expression of CERl gene in blackberry was analyzed by qRT-PCR. The expression of CER1 in blackberry plantlet under different light conditions was analyzed by qRT-PCR. The aim of this study is to understand the characteristics of CERI in blackberry plants, and to provide a theoretical basis for the molecular mechanism research of blackberry wax synthesis.

\section{Materials and Methods}

\section{Materials}

In April of 2015, the young leaves and fruits of blackberry cultivar 'Arapaho', which are planted at the teaching and scientific research base of Sichuan Agricultural University were taken as materials and frozen in liquid nitrogen for RNA extraction. Fruits of three maturity level were included: young fruits (5 days post anthesis, DPA), red fruits (20 DPA), and black fruits (25 DPA). Fruits of the same developmental stage were randomly selected on different branches of the same shrub. 20 Fruits were randomly selected as one biological replicate. Three replicates were conducted for each stage.

The 'Arapaho' sterile seedlings were established from April 2015. Plantlets were subcultured every four weeks in MS medium supplemented with $20 \mathrm{~g} / \mathrm{L}$ sucrose. Plants were cultured in a standard tissue culture room with temperature $25{ }^{\circ} \mathrm{C} \pm 2{ }^{\circ} \mathrm{C}, 16 \mathrm{~h}$ light/day, $70 \%$ RH.

\section{Total RNA Extraction and Reverse Transcription}

The young leaves, green fruits, red fruits and black fruits were powdered in liquid nitrogen. Total RNA was extracted by modified CTAB method [9]. cDNA was synthesized according to the instructions of the Reverse Script kit Prime Script RT reagent Kit with gDNA Eraser (Takara, Dalian, China).

\section{CER1 Gene Cloning and Sequencing}

From the transcriptome data of our previous research (Bioproject PRJNA401210), a transcript homologous to Arabidopsis thaliana CER was extracted. Two specific primers were designed in Primer 5.0 software (CER1-F: GCG CAA CAG ATG ATT AAT TAA KCA GC and CER1-R: GAA AAA ATG GCC ACC ACT CCT GG, the underline is the start codon).

PCR amplification was conducted in $20 \mu \mathrm{L}$ reaction mixture, which $1 \mu \mathrm{L}$ the forward and reverse primer respectively, $1 \mu \mathrm{L}$ cDNA, $10 \mu \mathrm{L}$ Prime STAR Max DNA 
Polymerase (Takara, Dalian, China). The amplification conditions were listed as pre-denaturation at $94^{\circ} \mathrm{C}$ for $5 \mathrm{~min}$; denaturation at $94^{\circ} \mathrm{C}$ for $30 \mathrm{~s}$, annealing at $60^{\circ} \mathrm{C}$ for $30 \mathrm{~s}$, extension at $72^{\circ} \mathrm{C}$ for $2 \mathrm{~min}$, for a total of 30 cycles followed by a finally extension at $72{ }^{\circ} \mathrm{C}$ for $10 \mathrm{~min}$. PCR products were detected by $1 \%$ agarose gel electrophoresis. After the recovering from the gel, the specific PCR amplicon was ligated into pEASY-Blunt vector and transformed into E. coli Trans1-T1 (Transgen, Beijing, China). After overnight culture and single colonies were picked for detection. The positive clones were sent to Sangon Biotech for sequencing.

\section{Bioinformatic Analysis}

The open reading frame was searched with NCBI ORF finder. Protein molecular weight and theoretical isoelectric point were analyzed with ProtParam. Conserved domains were screened by NCBI-CDD. Multiple sequence alignments were performed with MAFT. Phylogenetic tree was constructed by IQTREE.

\section{Real-time Quantitative Analysis of CER1 Gene Expression}

The blackberry actin gene was used as an internal reference (Qactin-F: AGA CTA CAT ACA ACT CCA TCA T and Qactin-R: ACC AAT CCA GAC ACT ATA CTT). The RT-PCR primer was designed based on the sequenced CER1 gene sequence, Qcer-F: T GGT GGA TGG AAG TAG and Qcer-R: GGC GTA AGC AAC CTT TGT GAG.

The expression of CERl gene in different fruit maturity level and under different light irradiation conditions. Thirty-day old subculture seedlings of 'Arapohol' were placed under top red light $(660 \mathrm{~nm})$, blue light $(470 \mathrm{~nm})$, combinations of different quantity of the two (R-B 2:8, R-B 5:5, R-B 8:2) in a growth chamber which was equipped with LED. Light intensity was set to $5000 \mathrm{~lx}, 25^{\circ} \mathrm{C}, 16 \mathrm{~h} \mathrm{light/day}$. Plants grow under white light were used as controls. Leaves at the same node level were collected after $72 \mathrm{~h}$ of irradiation. Three replicates were set up for each treatment. Total RNA was extracted for analysis of the expression of CERl under such conditions.

RT-qPCR reaction mixture consists of SYBR Green fluorescent dye $10 \mu \mathrm{L}$, the upstream and downstream primers $0.8 \mu \mathrm{L}$, respectively, cDNA $1.5 \mu \mathrm{L}, \mathrm{ddH}_{2} \mathrm{O}$ to $20 \mu \mathrm{L}$. Reaction were conducted at $95^{\circ} \mathrm{C}$ for $3 \mathrm{~min}$, followed by 40 PCR cycles of $95^{\circ} \mathrm{C} 10 \mathrm{~s}$, $55^{\circ} \mathrm{C} 30 \mathrm{~s}, 72^{\circ} \mathrm{C} 15 \mathrm{~s}$. Fluorescence was collected at the third step of each cycle. Reactions with no template cDNA were used as negative controls. All PCR reactions were replicated three times, and the data were analyzed by $2^{-\Delta \Delta \mathrm{Ct}}$ method. The relative expression values were subjected to One-way ANOVA analysis in SPSS software (v20.0).

\section{Results}

\section{CER1 gene in Rubus Spp.}

The CER I gene homologous to Arabidopsis CER1 was successfully identified in Rubus spp. The open reading frame of this gene was $1878 \mathrm{bp}$, encoding a peptide of 625 amino acid residues with a molecular weight of $72.3 \mathrm{kD}$. Theoretical isoelectric point is 8.59. Multiple sequence alignment was conducted with the currently identified other CER1 proteins. The predominant various were observed at the $\mathrm{C}$ terminal (Fig. 1). 


Consensus
Identity
1. XM_021010907.1
2. XM_018987966.1
3. XM_016031007.1
4. XM_009378576.2
5. XM_008340198.2
6. XM_008234039.1
7. XM_004307233.2
8. RuCer1
9. XM_009588667.2
10. XM_006464299.1
11. XM_004976137.2
Consensus
Identity
1. XM_021010907.1
2. XM_018987966.1
3. XM_016031007.1
4. XM_009378576.2
5. XM_008340198.2
6. XM_-008234039.1
7. XM_004307233.2
8. RuCer1
9. XM_009588667.2
10. XM_006464299.1
11. XM_004976137.2

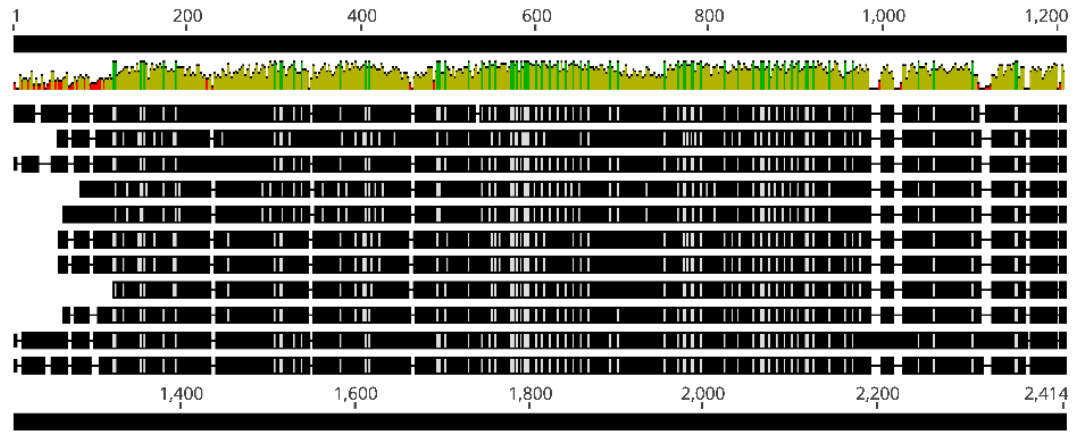

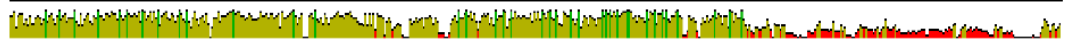



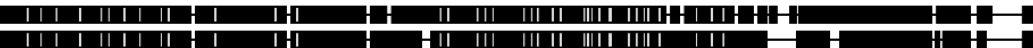
III III

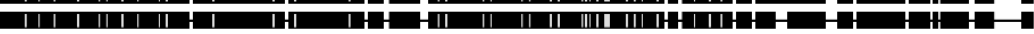
(1)

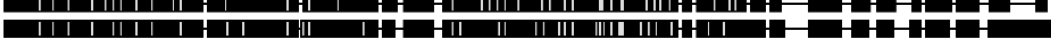

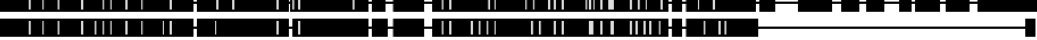

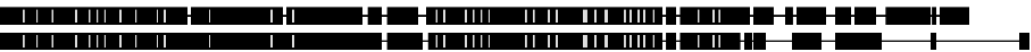

1. XM-004976137.2

Figure 1. Sequence alignment of CER1 related proteins. Conserved amino acids were marked in black, gaps were indicated as lines in Geneious software.

\section{Phylogeny of CER1}

The phylogeny of CER related proteins from species like Malus, Pyrus, Citrus were referred by most likelihood (ML) trees (Fig. 2). The blackberry CER1 protein has the highest similarity with the amino acid sequence of strawberry CER1 protein, indicating the closest evolutionary relationship with strawberry. Rosaceae species formed a unique clade, but can be grouped into two clusters with these species in this investigation.

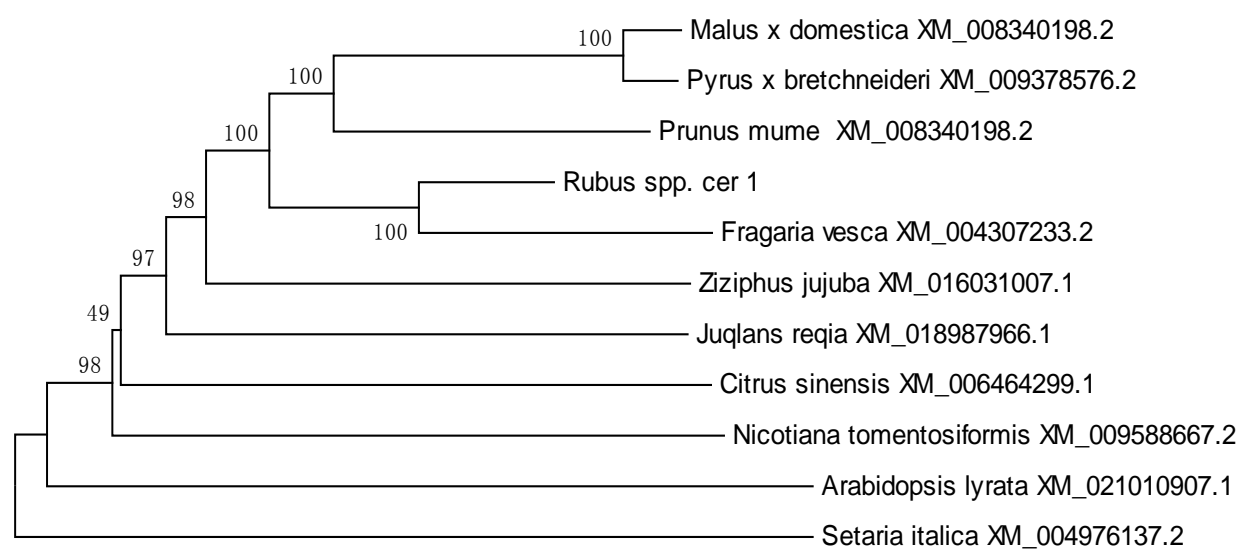

Figure 2. ML tree of CER1 of blackberry and other plants. The scale bar represents number of substitutions per site

\section{CER1 Gene Expression in Different Developmental Stages of Fruit}

The RT-qPCR was used to access the expression of CERl gene during blackberry fruit development. As shown in Fig. 3A, the expression level of CERl gene increased with fruit ripening. 


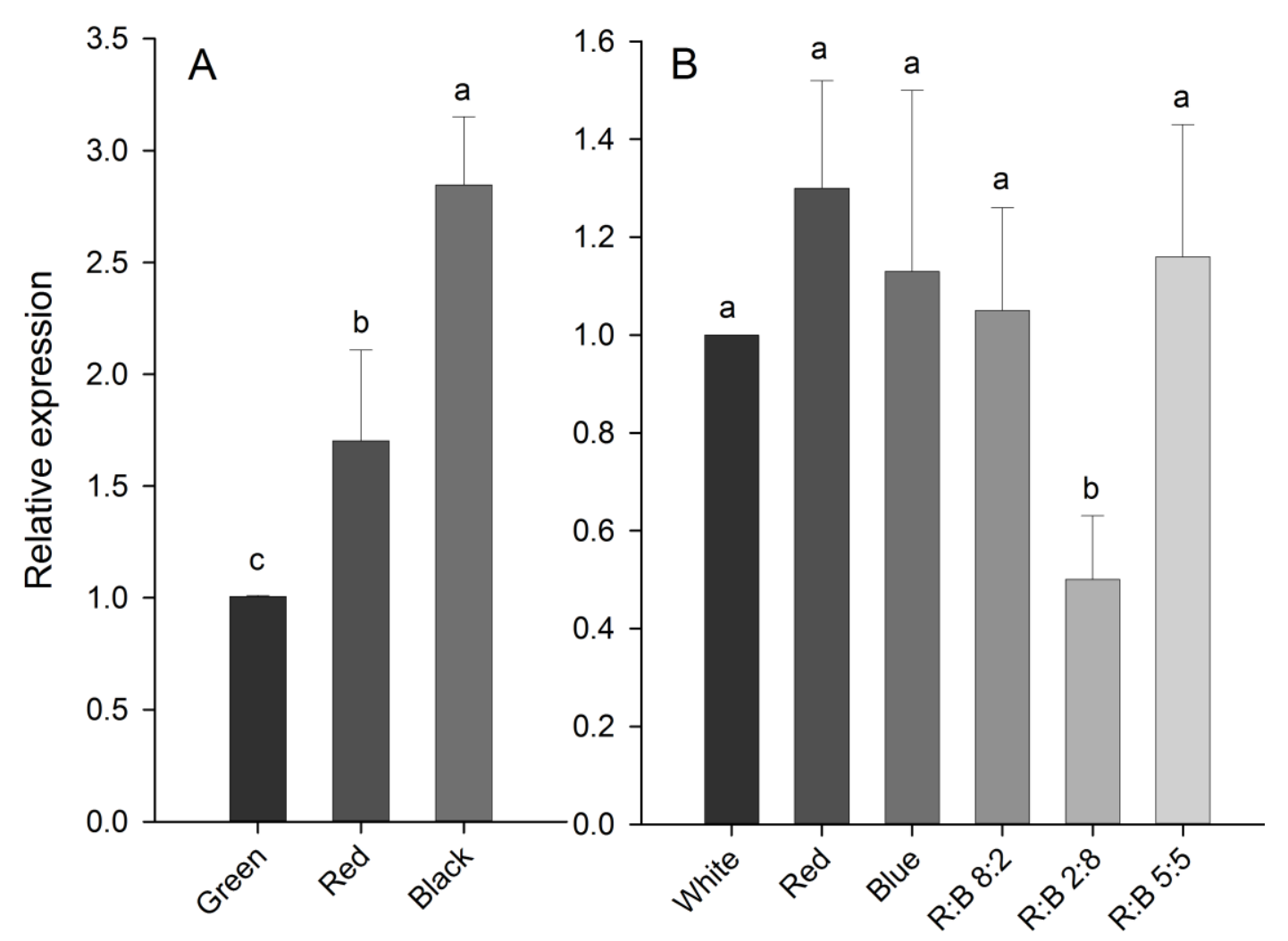

Figure 3. Expression profiling of CERl gene in different fruit development stages and responses to different light irradiation in blackberry leaves. Same letters indicate non-significant differences among stages (One-way ANOVA, p<0.05).

\section{Light-induced CER1 Expression}

The blackberry seedlings were treated with six different light conditions, respectively. The expression of CERl gene was analyzed by qRT-PCR (Fig. 3B). The results showed that there was no significant difference in the expression of CERl gene between the four treatments of full-red light, full-blue light and red-blue light at a ratio of 8:2 and red-blue light at a ratio of 5:5 when compared with white light. Obviously, with the increasing ratio of blue light, the $C E R I$ was significantly repressed. But this repression can be recovered with the continuing increasing (to 100\%).

\section{Discussion}

The blackberry CER 1 protein sequence obtained in this study has high similarity to CER1 protein sequences of other species. It has the closest evolutionary relationship with strawberry and contains conserved domains: fatty acid hydroxylase domain and WAX2-C domain. The results of RT-qPCR analysis showed that the expression level of CERI was increased with the maturation of fruit, indicating that the function of CER1 in blackberry may be similar to that of CER1 in Arabidopsis. Through the transformation of long-chain fatty acids for alkanes, to achieve plant epidermal wax accumulation, but its specific regulatory role and metabolic pathways, still need further verification. The fruits mature at a season when the light intensity continues growing. The increasing CERl expression might trigger the synthesis of wax, which can to some extent protect the fruits from injury. Studies have shown that the synthesis of plant epidermal wax is closely related to the external environment. The expression level of 
CER6 is increased under light-induced and osmotic stress. After $\mathrm{NaCl}$, drought and mannitol treatment, KCS, CER10, KCRI and CERI transcriptional levels increase but decrease in dark and low-temperature treatments, whereas CERl and CER5 genes overexpress drought-enhanced syntheses of alkanes [10]. In this study, blackberry seedlings were treated with six different light combinations respectively. With the increase of the ratio of red or blue light, the expression of $C E R l$ gene decreased first and then increased. At the time of the ratio of red and blue is 2:8, the expression of CERI was the lowest. This may be due to photoreceptors respond differently to red and blue light. However, its specific regulatory mechanism needs further study.

\section{Acknowledgement}

This research was financially supported by the National Natural Science Foundation of China (Grant Number 31600232).

\section{References}

[1] E.A. Fich, N.A. Segerson, J.K. Rose, The plant polyester cutin: Biosynthesis, structure, and biological roles, Annu. Rev. Plant Biol. 2016, 67(1), 207-233.

[2] D.K. Kosma, B. Bourdenx, A. Bernard A, The impact of water deficiency on leaf cuticle lipids of Arabidopsis, Plant Physiol. 2009, 151(4), 1918-1929.

[3] L. Samuels, L. Kunst, R. Jetter, Sealing plant surfaces: cuticular wax formation by epidermal cells, Annu. Rev. Plant Biol. 2008, 59(1), 683.

[4] Z. Wang, O. Guhling, R. Yao R, Two oxidosqualene cyclases responsible for biosynthesis of tomato fruit cuticular triterpenoids, Plant Physiol. 2011, 155(1), 540-551.

[5] B. Bourdenx, A. Bernard, F. Domergue, Overexpression of Arabidopsis ECERIFERUM1 promotes wax very-long-chain alkane biosynthesis and influences plant response to biotic and abiotic stresses, Plant Physiol. 2011, 156(1), 29-45.

[6] S. Lacey, K. Ljerka, J. Reinhard, Sealing plant surface: Cuticular wax formation by epidermial cells, Annu. Rev. Plant Biol. 2008, 59, 683-707.

[7] A. Bernard, F. Domergue, S. Pascal, Reconstitution of plant alkane biosynthesis in yeast demonstrates that Arabidopsis ECERIFERUM1 and ECERIFERUM3 are core components of a very-long-chain alkane synthesis complex, Plant Cell 2012, 24(7), 3106-3118.

[8] T.H. Yeats, J.K. Rose, The formation and function of plant cuticles, Plant Physiol. 2013, 163(1), 5-20.

[9] Q. Chen, H. Yu, X.R. Wang, An alternative cetyltrimethyl ammonium bromide-based protocol for RNA isolation from blackberry (Rubus L.), Genet. Mol. Res. 2012, 11(2), 1773-1782.

[10]J. Joubès, S. Raffaele, B. Bourdenx, The VLCFA elongase gene family in Arabidopsis thaliana: phylogenetic analysis, 3D modelling and expression profiling, Plant Mol. Biol. 2008, 67(5), 547-566. 